\title{
Research on Performance Evaluation Criteria for IP Multicast
}

\author{
Ningwei Sun ${ }^{a}$, Lu Wang ${ }^{b}$ and Yong Liu ${ }^{c}$ \\ Naval Academy of Armament, Beijing 100073, China \\ asunningwei@sina.cn, bjichenzhe@126.com, c31788772@qq.com
}

Keywords: IP Multicast, Performance Evaluation Criteria, Power Formula.

\begin{abstract}
Based on the power formula, this paper studies the performance standard of IP multicast performance evaluation. Firstly, the paper analyzes the performance target of IP multicast application. And then, two kinds of performance evaluation criteria were put forward, one of which is based on throughput (T) and Quality of Service (QoS) requirements, and another of which is based on QoS requirements and fairness $(\mathrm{F})$ requirements. Finally, the combination of using two kinds of performance evaluation criteria to evaluate the IP multicast network applications were discussed.
\end{abstract}

\section{Introduction}

This paper studies a basic problem of IP multicast performance evaluation, that is, with what kind of standards for performance evaluation. Performance evaluation criteria, which is based on the performance evaluation indicators, is to give an overall situation description of the network performance according to the value of different indicators.

At present, the research on network performance evaluation standard is focused on the level of network transmission control, and the Power formula describing the relationship between throughput and delay is proposed [1]. Two kinds of performance evaluation criteria are proposed in the reference 2 . In the first criteria, the Power formula is improved, with the multi-service competition mechanism introduced. And the second criteria is to introduce the principle of proportionality fairness, combined the fairness, delay and packet loss rate of resource allocation, and formulate comprehensive evaluation standard. The combination of the two types of standards is also discussed. In the reference 3, the performance evaluation indicators consisting of quantity and quality of service and fairness, performance evaluation time scale and evaluation granularity of service quality were all discussed. By defining the normalized performance function, packet loss rate, and forward delay, and other service quality and service quantity were all considered to evaluate the effectiveness of the network. To evaluate the fairness of the network, the relationship between random process mean and variance, and the improved formula of RajJain fair index were both used in the work.

So far, there is a lack of research on performance evaluation standards related to IP multicast. Based on the previous study on the evaluation index system of IP multicast performance [4], Section 2 analyzed the performance objectives of IP multicast applications. Section 3 examined the performance evaluation criteria of multicast performance as a whole. Section 4 and section 5 made a detailed study considering the throughput and quality of service requirements, the quality of service requirements and the fairness, by respectively putting forward performance evaluation criteria 1 and 2. And section 6 discussed the combinations of two criteria.

\section{Performance Objectives of IP Multicast Application}

Multicast application performance studies are usually considered to meet the objectives as follows:

(A)The overall efficiency of the network performance. That is, through the network load balancing situation, resource utilization, congestion and other aspects of the overall efficiency of the network to determine the level of performance advantages and disadvantages.

(B)Quality of service (QoS) requirements. That is, through the application of system bandwidth, delay and packet loss rate and other aspects of the application system to meet the quality of service requirements to determine the merits of performance. 
(C)Fairness. That is, under the premise of limited network resources, through the correlation between different applications, whether to isolate each other, resource allocation strategy and other aspects, whether different application systems are fair access to resources to determine the merits of performance.

The above three types of performance evaluation objectives are measured through the performance evaluation index system.

(A) Throughput (T) should be used to represent the overall efficiency of the network performance. A network node is usually described as of high performance if it has high throughput.

(B) Quality of service (QoS) requirements include bandwidth (Bandwidth, BDW), Forwarding Latency (FL), Packet Loss Rate (PLR) and Delay Jitter (Jitter, JIT), etc.

(C) Fairness (F) requirements are more complex. There is no standard way to measure fairness. Some studies regard all the network flow getting relatively fair resources as fairness. And other studies think that resources should be distributed based on the load situation.

\section{Performance Evaluation Criteria of IP Multicast}

Different performance evaluation indexes have different relationships, positive correlation, or negative correlation. Performance objectives cannot be optimal at the same time. It is a difficult question of how to meet these different objectives at the same time.

The main difficulties are: on the one hand, there are resource and policy constraints in the network, such as network bandwidth resources, processor speed and control strategy rationality; on the other hand it is difficult to meet the throughput, forwarding delay and packet loss rate, and sometimes these requirements even contradict each other. So trade off is needed to evaluate the performance of the various objectives.

According to the sensitive situation of multicast application with delay jitter, we take $\mathrm{T}$ to measure the overall efficiency of the network, and the FL, PLR, and JIT as the representative of QoS, to build the following two performance evaluation criteria.

(1) Throughput (T) + Quality of Service Requirements (QoS): These two types of indicators are actually a pair of contradictions. T can be improved by letting as much as possible packets go through the network, meanwhile as the number of packets on the network increased, the length of each router waiting queue Increase, the FL will also increase. Similarly, if too much packets on the network, the PLR and JIT will both increase.

(2) Quality of Service Requirements (QoS) + fairness (F): FL + F, using queuing delay as a criterion for fairness allocation. PLR $+\mathrm{F}$, using packet loss rate (PLR) as a criterion for fairness allocation. JIT + $\mathrm{F}$, using packet loss rate (PLR) as a criterion for fairness allocation.

\section{Performance Evaluation Criteria 1: Throughput $\mathbf{T}+$ Quality of Service Requirements QoS}

Reference 6 proposed a Power Formula for a network that describes the effectiveness of resource allocation strategies by the relationship between throughput and delay:

$$
\text { Power }=\frac{\text { Throughput }^{\alpha}}{\text { Delay }}(0<\alpha<1) .
$$

Reference 2 enhanced the Power Formula by considering the relationship between throughput and delay in the case of multi-service model. The Power Formula under multi-queue M / M / n model is proposed:

$$
\text { Power }=\frac{\text { Throughput }^{\alpha}}{\bar{d}[\lambda]}=\frac{\left[\sum T_{i}\right]^{\alpha}}{\frac{1}{\lambda} \sum \lambda_{i} \bar{d}_{i}}(0<\alpha<1) .
$$

In the above formula, $\lambda_{i}$ represents the average arrival rate of the service class $i, \bar{d}_{i}$ represents the average queuing delay of the service class $i$, the total average arrival rate of the service is $\lambda=\sum \lambda_{i}$. 
So, The contribution rate of the service class $i$ to the system average delay is $\frac{\lambda_{i}}{\lambda} \bar{d}_{i}$. And the total average delay of the system is $\bar{d}[\lambda]=\frac{1}{\lambda} \sum \lambda_{i} \bar{d}_{i}$.

\subsection{Throughput(T) + Forward Latency $($ FL)}

IP multicast application is a typical multi-service model. The same multicast source corresponds to multiple multicast data receivers, so it conforms to multi-queue M / M / $\mathrm{n}$ model. In the study of multicast network performance evaluation, we focus on the contribution of FL to the overall delay (D) of the network. Therefore, the Power Formula in M / M / $\mathrm{n}$ model is improved. In the case of multicast application, with $\mathrm{T}$ and FL as two types of performance evaluation indicators, we describe the effectiveness of the network performance evaluation criteria as bellows.

Definition 5.1 (Power Formula for multicast T and FL):

$$
\text { Power }=\frac{T^{\alpha}}{\overline{f l}[\lambda]}=\frac{\left[\sum T_{i}\right]^{\alpha}}{\frac{1}{\lambda} \sum \lambda_{i} \overline{f l_{i}}}(0<\alpha<1) \text {. }
$$

In the above formula, $\lambda_{i}$ represents the average arrival rate of the $i$ multicast receiver, and $\overline{f l_{i}}$ represents the average forwarding delay of the $i$ multicast receiver, and $\lambda=\sum \lambda_{i}$ represents the total average arrival rate of the system. So, the contribution of the $i$ multicast receiver to the average delay of the system is $\frac{\lambda_{i}}{\lambda} \overline{f l}_{i}$, and the total system average delay is $\overline{f l}[\lambda]=\frac{1}{\lambda} \sum \lambda_{i} \overline{f l}_{i}$.

Using AML (average multicast forwarding latency) represents the average multicast forwarding delay, the above formula can be expressed as:

$$
\text { Power }=\frac{T^{\alpha}}{\overline{f l}[\lambda]}=\frac{\left[\sum T_{i}\right]^{\alpha}}{\frac{1}{\lambda} \sum \lambda_{i} \overline{f l}_{i}}=\frac{\left[\sum T_{i}\right]^{\alpha}}{\sum a m l_{i}}(0<\alpha<1)
$$

\subsection{Throughput(T) + Packet loss rate(PLR)}

According to the research results of reference 2, Power Formula of T and PLR is the same as the Power Formula of $\mathrm{T}$ and FL. Therefore, through $\mathrm{T}$ and PLR two types of performance evaluation indicators, we can also describe the effectiveness of network performance evaluation.

Definition 5.2 (Power Formula for multicast T and PLR):

$$
\text { Power }=\frac{T^{\alpha}}{\overline{p l r}[\lambda]}=\frac{\left[\sum T_{i}\right]^{\alpha}}{\frac{1}{\lambda} \sum \lambda_{i} \overline{p l r}_{i}}(0<\alpha<1) \text {. }
$$

In the above formula, $\lambda_{i}$ represents the average packet arrival rate of the $i$ multicast receiver, $\overline{p l r} r_{i}$ represents the average packet loss rate of the $i$ multicast receiver, $\lambda=\sum \lambda_{i}$ represents the total average arrival rate of the system. So, the contribution of the $i$ multicast receiver to the average packet loss rate of the system is $\frac{\lambda_{i}}{\lambda} \overline{p l r_{i}}$, and the total system average packet loss rate is $\overline{p l r}[\lambda]=\frac{1}{\lambda} \sum \lambda_{i} \overline{p l r_{i}}$.

\subsection{Throughput(T) + Delay Jitter(JIT)}

The delay jitter of the $i$ multicast receiver can be calculated by MaxML (maxnimum transmission delay) and MinML (minimum transmission delay):

$j i t_{i}=\max m l_{i}-\min m l_{i}$

Assuming that the minimum propagation delay of the $i$ multicast receiver is a nonzero minimum $\min m l_{i}=\varepsilon_{i}$,

then, 
$\max m l_{i}=j i t_{i}+\min m l_{i}=j i t_{i}+\varepsilon_{i}$,

and,

$\frac{\lambda_{i}}{\lambda} \overline{f l}_{i}=a m l_{i}=\frac{\max m l_{i}+\min m l_{i}}{2}$,

thus,

$\frac{\lambda_{i}}{\lambda} \overline{f l}_{i}=\frac{\max m l_{i}+\min m l_{i}}{2}=\frac{j i t_{i}+\varepsilon_{i}+\varepsilon_{i}}{2}=\frac{j i t_{i}}{2}+\varepsilon_{i}$,

So,

$\overline{f l}[\lambda]=\frac{1}{\lambda} \sum \lambda_{i} \overline{f l}=\sum\left(\frac{j i t_{i}}{2}+\varepsilon_{i}\right)$.

Definition 5.3 (Power Formula for multicast T and JIT):

Power $=\frac{T^{\alpha}}{\overline{f l}[\lambda]}=\frac{\left[\sum T_{i}\right]^{\alpha}}{\sum\left(\frac{j i t_{i}}{2}+\varepsilon_{i}\right)}\left(0<\alpha<1, \varepsilon_{i} \approx 0\right)$.

In the above formula, $j i t_{i}$ represents the delay jitter the $i$ multicast receiver, $\varepsilon_{i}$ represents the minimum forwarding delay of the $i$ multicast receiver, and $\varepsilon_{i}$ is a non-zero minimum value. If an approximate value is taken, then:

$$
\text { Power }=\frac{\left[\sum T_{i}\right]^{\alpha}}{\frac{1}{2} \sum \text { jit }_{i}}(0<\alpha<1) .
$$

\section{Performance Evaluation Criteria 2: Quality of service requirements QoS + fairness F}

\subsection{Basic Assumptions of Fairness Considerations}

There is a basic assumption when using fairness as a performance evaluation standard, which is, the multicast network is in a resource-limited and of heavy load condition, and there is no strict distinction between service quality control for network multicast applications.

Assumption 1: Proportional Fairness Assumption

Considering the fairness performance evaluation standard and referring to the relevant ideas put forward in the reference 2 and 7, the network resource is distributed according to the service performance of different service class performance. That is, the ratio of the actual performance among different service applications in the network is equal to the ratio of the performance requirements of the different service classes, and the performance requirements of the different service classes are measured with the quality of service distinction.

Assuming $c 1<c 2<\cdots<c N$ is the quality of service distinction of $\mathrm{N}$ services, $q_{i}$ is differentiated by the value of the service class $i$, the proportional fairness assumption can be expressed as:

$$
\frac{q_{i}}{q_{j}}=\frac{c_{i}}{c_{j}}(i, j=1,2, \cdots N) \text {. }
$$

As can be seen from the above equation, even if the quality of service of each service class changes with the respective load changes, the service quality ratio between different service classes does not change.

The proportional fairness assumption requires the value of the performance evaluation index can be controlled by transmission control. Otherwise, if the performance evaluation index cannot be controlled, it is difficult to ensure that the proportional equation is established.

Assumption 2: Network Resource Limited and of Heavy Load Assumption

Because if the network resources are sufficient, according to the network resources in a light load situation, all network applications are able to meet the requirements of quality of service.

Assumption 3: No Strict Distinction Between Quality of Service Control 
If strict quality control is achieved, high-priority network applications are always given priority, and low-priority network applications are always met after. There is no question of fairness.

Assumptions 4: Service Performance Classification

In the fairness study, the different types of applications carried on the network are classified into several service classes according to the service performance requirements. According to the actual characteristics, the application on the network is divided into the following services:

(a) Unicast service class, as the background of the flow of this multicast study to be considered, with letters $m$;

(b) Real-time interactive multicast service class, with letters $n$,

(c) Real-time non-interactive multicast service class, with letters $p$;

(d) Non-real-time multicast service class, with letters $q$.

\subsection{Forward Latency(FL) + Fairness $($ F $)$}

For the above four service classes $m, n, p, q$, using a time period of the average forwarding delay $\overline{f l_{m}}, \overline{f l_{n}}, \overline{f l_{p}}, \overline{f l_{q}}$ to represent the average performance of each service class, in where the average multicast forwarding delay, refers to for a class of application in a period of time of all the multicast data receivers, rather than the forwarding delay for a particular multicast data receiver. And then:

$$
F=\frac{\overline{f l_{m}}}{\delta_{m}}=\frac{\overline{f l_{n}}}{\delta_{n}}=\frac{\overline{f l_{p}}}{\delta_{p}}=\frac{\overline{f l_{q}}}{\delta_{q}} .
$$

The parameters $\left\{\delta_{m}, \delta_{n}, \delta_{p}, \delta_{q}\right\}$ are the service quality requirements of the service class, which is called the delay distinction parameter.

The above equation considers the average forwarding delay in a period of time. In fact, since the network has more network traffic mutations, the forwarding delay still needs to be considered in the shorter time interval.

The specific solution is that, with $\overline{f l}(t, t+\Delta t)(i=m, n, p, q)$ represents the average delay of a service class, in a very short time interval $(t, t+\Delta t)$, the formula for proportional fairness becomes:

$$
F=\frac{\overline{f l_{m}}(t, t+\Delta t)}{\delta_{m}}=\frac{\overline{f l_{n}}(t, t+\Delta t)}{\delta_{n}}=\frac{\overline{f l_{p}}(t, t+\Delta t)}{\delta_{p}}=\frac{\overline{f l_{q}}(t, t+\Delta t)}{\delta_{q}} .
$$

The above formula should be considered, just in the time interval $(t, t+\Delta t)$, four service classes $m, n, p, q$ have data packets through the network, if a service class does not have a packet, $\overline{f l}_{i}(t, t+\Delta t)(i=m, n, p, q)$ should be regarded as undefined, regardless of the proportion of fairness.

\subsection{Packet loss rate(PLR) + fairness $(F)$}

When the PLR is used as a measure of the proportion of fairness, there is less case of burst traffic. Because the PLR itself is a statistical indicator over a period of time. The average PLR here refers to the total packet loss rate of all multicast data receivers in a certain period of time. Rather than the packet loss rate for a particular multicast data receiver. Using $\overline{p l r_{m}}, \overline{p l r_{n}}, \overline{p l r_{p}}, \overline{p l r_{q}}$ represent the average packet loss rate of the four service classes. There are:

$$
F=\frac{\overline{p l r_{m}}}{\sigma_{m}}=\frac{\overline{p l r_{n}}}{\sigma_{n}}=\frac{\overline{p l r_{p}}}{\sigma_{p}}=\frac{\overline{p l r_{q}}}{\sigma_{q}} .
$$

The parameters $\left\{\sigma_{m}, \sigma_{n}, \sigma_{p}, \sigma_{q}\right\}$ are the service quality requirements of the service class, which is called the packet loss rate distinction parameter.

\subsection{Delayed Jitter(JIT) + Fairness(F)}

It is difficult to achieve the sufficient condition of the proportional fairness assumption because it can not control the JIT. Therefore, as an open topic, this paper does not carry on the delay jitter and fairness as the performance evaluation criteria. 


\section{Combination of Two Performance Evaluation Criteria}

In the reference 2 , it is proved that the performance evaluation criteria 1 and criteria 2 are independent of each other. That is, for the proportion of fairness of criteria 2, will not affect the criteria 1 of indicators value. Therefore, two criteria can be combined together as a network multicast performance evaluation standard.

Criteria 1 is concerned with the impact of transmission control strategies on the overall performance and quality of service requirements of networks and systems. Criteria 2 focuses on user requirements and fairness between multiple competing service classes where network resources are limited.

\section{Summary}

This paper studies the evaluation standard of network IP multicast performance. Based on the performance evaluation indicators, this paper analyzed the performance objectives of different application types, and put forward the performance evaluation criteria such as integrated throughput, application QoS, system fairness.

The main contributions of this chapter are as follows:

(A) For the multicast application, the classical network Power Formula is improved to make it suitable for the performance evaluation of the multicast network, which has a greater scope of application and put forward the performance evaluation standard which can be used to distinguish the application type.

(B)Considering the performance requirements of different application types, the practical characteristics of multicast applications and the fairness of multicast applications, the evaluation criteria of fairness evaluation of multicast application performance evaluation are proposed.

(C) The two performance evaluation criteria are combined to form a performance evaluation standard, which can be used for multiple performance evaluation indicators, and also can be used for practical performance evaluation.

Some basic assumptions are made for the study in this paper, which are in line with the actual situation of network IP multicast applications. And there are still some open topics required further research, such as the research on the more general performance evaluation criteria, and the optimization or comparison of different standards for multicast network performance evaluation.

\section{References}

[1]. Peterson L L, Davie B S. Computer Networks: A System Approach. Second Edition. Morgan Kaufmann Publisher, 2000, 454-457.

[2]. Jiang Yong, Lin Chuang, Wu Jian-Ping. Integrated Performance Evaluation Criteria for Network Traffic Control. Chinese J. Computers, 2002(8):869-877.(In Chinese)

[3]. Lin Chuang, Zhou Wen-Jiang, Tian Li-Qin. Research on Performance Evaluation Criteria for IP Network Traffic Control. Acta Electronica Sinica, 2002(12A):1973-1977. (In Chinese)

[4]. Sun Ning-wei, Liu Yong, Zhao Yu, et al. Survey of Methods for Performance Evaluation of IP Multicast. Application Research of Computers, 2016(33):15-17. (In Chinese)

[5]. Lin Chuan, Shi Xiaoqiu, Hu Bo, et al. Network Performance Testing and Analysis. BeiJing: Higher Education Press, 2009.9. (In Chinese)

[6]. Peterson L L, Davie B S . Computer Networks: A System Approach.Second Edition. Morgan Kaufmann Publisher, 2000.454-457.

[7]. Dovrolis $\mathrm{C}$ et al. Proportional differentiated services: Delay differentiation and packet scheduling. In: Proc SIGCOMM'99, Boston, USA, 1999. 109-120.

[8]. Liu Yong, Sun Ning-wei. Research on Muasure Methods for Performance Evaluation Metrics of IP Multicast. Network Security Technology and Application, 2017(3). (In Chinese)

[9]. Lin Chuang. Performance Evaluation of Computer Networks and Computer Systems. Tsinghua University Press, 2001(4). (In Chinese) 
[10]. Chen Jin, Wu Wenjun. Methods and Tools to Examine Multicast Traffic Performance. Computer Science, 2001,28(3):31-35.

[11]. Lin Chuang, Li Ya-juan, Wang Zhong-min. Status and Development of Formal Methods for Performance Evaluation. Acta Electronica Sinica, 2002,30(12A):1917-1922. (In Chinese)

[12]. Lin Chuang, Wu Jianping, Wang Dingxing. Stochastic High-level Petri Nets for Modeling and Performance Evaluation of a Transport Protocol. Journal of China Institute of Communications, 1994,15(6):9-16. (In Chinese)

[13]. Du Keliang, Zhang Xuan, Li Xing, et al. Birth-death model of IP multicast group behavior. J. Tsinghua University (Sci \& Tech), 2004, 44(1):134-137. (In Chinese)

[14]. Zhang Wenjie, Qian Depei, Bai Yuebin, et al. A Framework for Network Performance Metrics Definition. Computer Engineering and Applications, 2003,39(10):14-21. (In Chinese)

[15]. K. Dubray. Terminology for IP Multicast Benchmarking, Request for Comments: 2432, October 1998.

[16]. Request for Comments: 3918(RFC3918).Methodology for IP Multicast Benchmarking, April 2004.

[17]. KRZYSZTOF STACHOWIAK, TYTUS PAWLAK, MACIEJ PIECHOWIAK. Performance Evaluation of Multicast Overlay Routing Protocols. Image Processing \& Communication, vol. 17, no. 1-2, pp. 19-32. DOI: 10.2478/v10248-012-0012-4.

[18]. Stefan Egger and Torsten Braun. Performance Evaluation of Multicast for Small Conferences. Conference Paper in Lecture Notes in Computer Science, January 2003.

[19]. M.Nagaratna, V. Kamakshi Prasad, Raghavendra Rao. Performance Evaluation of Mesh-Based Multicast Routing Protocols in MANET's. International Journal of Advanced Computer Science and Applications. Vol.2, No.7, 2011, pp.44-52.

[20]. Takeshi Takahashi, Miikka Tammi, Heikki Vatiainen, Rami Lehtonen, Jarmo Harju. Implementation and Performance Evaluation of Multicast Control Protocol.

[21]. Xu Zhaofeng. Reaserch and Partial Implementation of Multicast Monitoring and Management System for Next-Generation Internet. Beijing University of Posts and Telecommunications, 2006. (In Chinese)

[22]. Wang Dongping. The design and implementation of a SLA-based multicast performance monitor system [D]. Southeast University, 2004. (In Chinese)

[23]. Sarac K, Almeroth K, Supporting Multicast Deployment Efforts: A Survey of Tools for Multicast Monitoring [Z], Mar.2001, 2-4. 